Caligrama, Belo Horizonte, v. 25, n. 3, p. 183-200, 2020

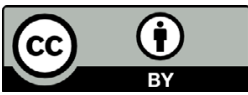

\title{
Mur Méditerranée de Louis-Philippe Dalembert : une réécriture transculturelle du topos de la traversée maritime, au prisme de la crise migratoire
}

\section{Mur Méditerranée by Louis-Philippe Dalembert : A Transcultural Rewriting of the topos of the Sea Crossing, through the Prism of the Migratory Crisis}

\author{
Lila Lamrous \\ Université Clermont Auvergne (UCA), Clermont-Ferrand / França \\ lila.lamrous@uca.fr \\ http ://orcid.org/0000-0002-0150-7722
}

Résumé : Mur Méditerranée de Louis-Philippe Dalembert est bâti sur le topos de la traversée maritime, interdite et périlleuse, effectuée par trois personnages féminins. Le topos place ce roman au cœur d'un ensemble de textes et requiert une lecture comparatiste. Il révèle alors une structure extrêmement dynamique, narrative et argumentative, qui accueille les échos des autres traversées collectives (exode du peuple juif, traite négrière, exil haïtien) offrant une variante au vagabondage de l'auteur haïtien. La réécriture transculturelle de ce motif permet de questionner la crise migratoire contemporaine tout en redéfinissant le sens de l'exil dans la mondialisation.

Mots clés : exil et traversée Mtime ; topos littéraire ; réécriture transculturelle ; migration féminine ; frontières.

Abstract : Mur Méditerranée by Louis-Philippe Dalembert is built on the topos of the forbidden and perilous maritime crossing, carried out by three female characters. The topos places this novel at the heart of a set of texts and requires comparative reading. It then reveals an extremely dynamic, narrative and argumentative structure, which receives the echoes of other collective crossings (exodus of the Jewish people, slave trade, Haitian exile) offering a variant to the "vagabondage" of the Haitian author. The transcultural rewriting of this motif allows us to question the contemporary migration crisis while redefining the meaning of exile.

Keywords: exile and sea crossing; literary topos; transcultural rewriting; female migration; borders. 
La figure du migrant en quête d'un refuge et d'une nouvelle vie semble produire un renouvellement de la problématique de l'exil (NOUSS, 2015), ce que l'on perçoit à travers plusieurs fictions romanesques récentes du champ éditorial français de même que dans différentes aires culturelles et linguistiques. Elle essaime notamment dans une production francophone, constituée autant de textes littéraires que de témoignages et d'essais, et qui se caractérise par son caractère transnational et transculturel, ainsi que le montre l'étude de Catherine Mazauric (2012). L'abondance et la constance de cette production (parallèle, du reste, au développement de la crise migratoire que connaissent le monde en général et l'Europe en particulier dans son rapport à l'Afrique ou à ses anciennes colonies), semblent indiquer que les frontières en tant qu'institutions créées arbitrairement par les hommes comme espace de restriction et d'assignation, inviolables et indépassables, disparaissent pourtant dans la création littéraire et dans l'imaginaire. Dalembert, obsédé par les partances dans sa vie personnelle comme dans son projet littéraire qui témoigne de son souci de faire tomber les frontières et de concevoir les rapports humains dans le sens du partage, inscrit son dernier roman dans ce contexte littéraire et géopolitique : Mur Méditerranée (2019) relate l'odyssée de trois femmes, en exil entre l'Afrique, le Moyen-Orient et l'Europe pour concrétiser leur rêve d'un ailleurs meilleur. Cette fiction puise explicitement dans l'actualité du phénomène migratoire du XXI siècle puisque Dalembert s'inspire d'un fait réel et documenté : le naufrage d'un navire en provenance de Libye vers Lampedusa en juillet 2014. L'actualité offre une trame et des éléments diégétiques au roman de Dalembert qui retrace avec réalisme et émotion la traversée maritime tragique de ces migrants dont les espérances se heurtent aux murs de la forteresse Europe, " forteresse inexpugnable, pareille au mur de glace de Game of Thrones » (DALEMBERT, 2019, p. 29).

La thématique de la migration clandestine n'est pas nouvelle dans l'œuvre de Dalembert qui l'a traitée, par exemple, dans L'autre Face de la mer (1998) en lien avec l'histoire d'Haïti marquée par la tragédie des boat-people dans les années 1970-80. L'auteur haïtien lui consacre également un roman éloigné de ses origines et de son inspiration haïtienne dans Noires Blessures (2010). Ce texte consacré au passage clandestin en Afrique montre l'enlisement, dans les sables du désert, du personnage principal Mamad contraint de renoncer au rêve d'échapper à son destin précaire. Il constitue surtout une réflexion sur la migration et le racisme, 
et nous intéresse ici en ce qu'il témoigne de la volonté de l'auteur de construire une réflexion autour de cette thématique et de mener son lecteur vers une prise de conscience du drame humain que constitue le périple des clandestins. On peut dire, en fait, que la thématique migratoire s'intègre naturellement dans la mythologie de l'auteur dont les personnages, voyageurs à son image, vouent une fascination pour l'océan et, surtout, cette " autre face de la mer», avec l'idée que " chaque mer a une autre rive et j'y arriverai » comme l'indique la citation de Pavese mise en exergue de Mur Méditerranée pour souligner ce désir de l'ailleurs et de la traversée. L'œuvre de Dalembert nous offre, outre une représentation complexe des départs et exils, une diversité de profils des personnages en partance ou en désir de partance : Et le Soleil se souvient... (1989) propose la déclinaison de ces partances que développe toute l'œuvre de l'auteur haïtien :

Puis il y eut tous ces départs et tous ces renoncements : ceux qui sont partis et ceux qui sont restés. Ceux qui sont partis de n'avoir pu rester, ceux qui sont restés de n'avoir pu partir, ceux qui sont partis pour n'avoir pas osé rester, par peur de crever ou à la recherche du pain distrait; et ceux qui sont partis comme ça, pour partir, pour n'être plus là... (DALEMBERT, 1998, p. 7).

Toutes ces figures et situations romanesques contribuent à imposer l'image de " l'aède vagabond " qu'est Dalembert, selon la formule de Diard (2018, p. 1), qui “'bourlingue la vie » (DALEMBERT, 2007, p. 42).

En effet, l'itinéraire géographique de Dalembert se confond avec les problématiques et les caractéristiques formelles de son œuvre, son vagabondage existentiel devenant en quelque sorte un vagabondage littéraire qui privilégie l'hybridité générique, l'intertextualité, la plasticité du style et le contact fécond des langues, ainsi que le montre Alessia Vignoli (2016). L'aspect polymorphe de cette œuvre singulière provient ainsi d'une mise en relation du vagabondage de l'auteur, des problématiques liées à la mobilité humaine et de l'histoire d'Haïti (certains critiques avançant que tous les auteurs originaires de cette île verraient leurs productions nourries par l'exil).

Mais la migration clandestine ne présente pas les mêmes aspects que le vagabondage libre et euphorique que revendique Dalembert dans sa vie et dans ses autres œuvres. Celui-ci assume l'errance en lui enracinée, choisit de s'installer dans l'éphémère du voyage que l'image du vagabondage met en exergue. Il prend soin d'ailleurs de 
différencier l'idée de vagabondage de concepts tels que l'erranceliée, dit-il, dans mon esprit à la malédiction divine qui s'est abattue sur le peuple juif $[\ldots]$ - ou le nomadisme - associé à un héritage culturel donc subi, à mon sens, du moins dans la plupart des cas. Or le vagabondage, tel que je le conçois, est choix conscient, assumé, solaire. (GHINELLI, 2005, p. 129).

La crise migratoire contemporaine révèle un autre visage de l'exil : le départ n'est pas seulement voyage vers un ailleurs rêvé, mais périple périlleux vers l'inconnu, itinéraire semé d'embûches dont le terme peut être une mort sans sépulture ou un déni d'humanité. Ainsi, que l'exprime Chochana, l'une des héroïnes de Mur Méditerranée (DALAMBERT, 2019, p. 80) : " arrachée à la terre matricielle », " plus que vers l'inconnu, elle avait l'impression d'être bannie en partance pour l'exil ».

Si Mur Méditerranée s'inscrit dans la suite logique de la production de Dalembert dont le déplacement est un sujet récurrent et omniprésent, il a ceci de nouveau d'abord que ce qui intéresse l'auteur ce n'est pas l'aller-retour, le départ et l'arrivée qui créent le sentiment $\mathrm{d}$ " " enracinerrance » mais la traversée, maritime de surcroit, ce passage d'une rive à l'autre qui constitue l'étape décisive et ultime dans le parcours migratoire, en ce sens où elle est franchissement de la frontière et de l'interdit. La traversée de la Méditerranée dans un "bateaumonde avec des gens de langue, d'ethnie et de religion différentes » (DALEMBERT, 2019) ouvre une parenthèse dans laquelle l'individu n'est finalement pas en suspens ou en attente d'un destin mais mobilisé pleinement dans le désir de se construire ailleurs (et ce désir prend la forme d'un combat intérieur avec ses propres démons et avec les forces extérieures autant naturelles que sociales). En cela, Dalembert renouvelle son traitement de la thématique de l'exil et réactive le topos narratif de la traversée martine, l'un et l'autre participant d'une réflexion plus large sur "l'expérience exilique » du migrant, laquelle est " condition et conscience » (NOUSS, 2015, p. 12-13). Son roman s'inscrit, en outre, dans un ensemble littéraire qui dépasse le corpus haïtien et crée des ponts entre des œuvres aux ancrages culturels et géographiques divers. Dalembert explore une thématique " rhizomatique », si l'on ose dire, en ce sens où elle est innervée d'échos multiples à d'autres textes, nourrie de l'Histoire, des mythes et des discours religieux d'origines variées. Enfin, cette mobilité est vue sous l'angle de la femme, à la fois actrice principale de la diégèse et focale quasi exclusive de la narration. C'est donc la 
traversée maritime comme topos et variante au vagabondage que nous nous proposons d' examiner, le topos narratif étant entendu comme "une configuration narrative récurrente d'éléments pertinents, thématiques ou formels » (WEIL, 2001) qui possède une valeur argumentative. Nous analyserons en particulier la perspective féminine choisie par Dalembert pour réécrire le topos, de même que la dynamique de la structure qui permet à l'auteur de produire une œuvre tout à la fois poétique et engagée, reflet d'une " culture transnationale » (MAZAURIC, 2012, p. 350).

\section{Une traversée au féminin}

S'inspirant d'un fait réel, Mur Méditerranée retrace, du 14 au 16 juillet 2014, une traversée clandestine à bord d'un chalutier usé, puis le sauvetage des migrants en perdition par le tanker danois TormLotte. Parce que l'Europe s'est habituée aux images des “ bateaux ivres » (MARI, 2015) que les médias dévoilent, la littérature se doit de redonner un nom, un visage, des pensées et des émotions, une humanité en somme, à ces naufragés que l'on semble dissimuler derrière l'étiquette " migrant » et des chiffres invérifiables. C'est bien d'abord le dessein de Dalembert qui s'intéresse au destin de trois femmes saisi au moment où elles attendent la traversée puis pendant la traversée maritime et où elles surmontent les épreuves en gardant " l'esprit tourné vers cet ailleurs où elles espéraient enraciner leurs rêves » (p2019, p. 80). Chochana (une Nigérianne chrétienne), Semhar (une Erythréenne juive) et Dima (une Syrienne musulmane) sont " entre cale et pont " (DALEMBERT, 2019, p. 86), comme les 750 autres passagers, " répartis en fonction du prix payé pour la traversée » (DALEMBERT, 2019, p. 86) mais réunies par le même projet de franchissement de la frontière interdite.

Ces trois figures sur lesquelles se concentre le récit permettent à l'auteur tout à la fois de placer le lecteur devant un drame collectif et des trajectoires très individualisées. Dès l'incipit, la diégèse nous fait passer du collectif " des centaines de damnés de la terre africaine » (DALEMBERT, 2019, p. 179) à l'individuel des espérances et luttes de trois femmes différentes par leurs origines, leur religion et leur histoire personnelle. La narration, parallèlement, construit des gros plans sur les passagers de la cale et du pont et des plans rapprochés qui mettent en lumière jusqu'aux pensées les plus intimes du trio féminin. Ainsi, dès Sabratha en Libye, 
Toute l'Afrique subsaharienne était représentée dans le hangar. Dans sa dislocation comme dans son humanité. Dans sa diversité et dans sa jeunesse. Les plus âgées parmi ces filles à la dérive dépassaient à peine la trentaine, observait Chochana, [...]. Plus d'une essuyait toujours les offenses acnéiques de l'adolescence. Plusieurs traînaient une grossesse amassée au cours de leur errance. Si Rachel et elle fuyaient une terre de moins en moins nourricière, une nature dont les mamelles s'étaient asséchées telle la main de Jéroboam, d'autres tentaient d'échapper à la guerre. Telles autres encore à une dictature qui interdisait à leur jeunesse de rêver d'avenir. C'était le cas de Semhar, une Erythréenne si frêle qu'on n'osait la toucher, de peur de la casser. (DALAMBERT, 2019, p. 67).

L'alternance des indéfinis pluriels et des prénoms crée ce vaet-vient entre une communauté de destin et des destins singuliers, l'amplitude de la représentation du drame collectif n'empêchant pas une attention aux différences car, ainsi que le note le narrateur, " les détresses, les parcours, les espoirs étaient les mêmes et tellement distincts » (DALEMBERT, 2019, p. 67).

La particularité de la représentation de la traversée clandestine que propose Dalembert réside également dans le fait qu'il la féminise. Aujourd'hui encore, peu d'études scientifiques et de textes littéraires sont consacrés aux femmes migrantes et, notamment, à celles qui sont jetées, souvent malgré elles, sur les routes de la migration clandestine. Le corpus littéraire examiné par Catherine Mazauric dans Mobilités d'Afrique en Europe, récits et figures de l'aventure fait ressortir, par exemple, les portraits très “ masculins » du harraga maghrébin et de " l'aventurier » subsaharien (MAZAURIC, 2012, p. 73-98) ; rares sont les textes de la force littéraire de celui de Marie Ndiaye, Trois Femmes puissantes (2010), tout entier consacré à l'itinéraire de cette femme puissante qu'est Khady Demba, qui au terme de son périple, fait de violences subies et d'humiliation, découvre "qu'elle était indivisible et précieuse, et qu'elle ne pouvait être qu'elle-même [...] et pensait également avec cette impondérable fierté, cette assurance discrète et inébranlable : je suis moi, Khady Demba » (NDIAYE, 2010, p. 296). Pour ce qui est de Fatou Diome, si son roman Le Ventre de l'Atlantique (2003) permet de cerner les contours d'une écriture féminine " migrante », son récent récit de la migration clandestine présente surtout les femmes comme étant "celles 
qui attendent »(DIOME, 2010). Catherine Mazauric (2014) conclut, de l'examen d'un corpus romanesque produit par des romancières de l'Afrique de l'Ouest, que leur représentation de la migration traduit surtout leur lutte pour une émancipation qui se manifeste dans la conquête de l'écriture.

Dalembert, à son tour, donne un visage féminin à cette quête de l'ailleurs dont l'une des spécificités du parcours semble être la violence sous toutes ses formes, le viol et l'esclavage sexuel en étant les formes les plus brutales et aliénantes. Le texte n'a de cesse de nous montrer dans " la masse de corps enchevêtrés dans une poignante pagaille ", "les visages déformés par les brimades et les privations quotidiennes " de cette communauté de filles reléguées au rang de "sacs de merde » (DALEMBERT, 2019, p. 111) par leurs geôliers. De même, nous entendons " les gémissements déchirants des filles dans la nuit " (DALEMBERT, 2019, p. 68), l'auteur nous laissant deviner les sévices endurés plutôt que de nous les peindre de façon crue ou détaillée. Les situations concrètes de violence infligée aux femmes sont nombreuses : précarité matérielle, soif, faim, maladie, violence des passeurs, viols et travaux forcés augmentent l'angoisse liés aux aléas du trajet, l'incertitude quant à la réussite du projet d'exil. La violence est partout sur ce trajet de femmes migrantes, comme si ce périple clandestin était surtout une succession d'épreuves génératrices de violences psychologiques et de traumatismes. Pourtant, ce sont ces épreuves qui permettent à Chochana, Semhar et Dima de se révéler à elles-mêmes, de découvrir leurs forces cachées, l'étape de la traversée maritime, en particulier, agissant comme un révélateur de soi. Toutes sont des femmes dont la détermination sans faille, la dignité et l'incompréhensible obstination font osciller le lecteur entre admiration et inquiétude. Elles semblent puiser leurs forces dans les profondeurs de leur malheur, dans le lien sororal qui s'établit entre Chochana et Semhar et, au moment du plus grand dénuement et du plus grand danger, elles trouvent leur richesse en elles-mêmes et, en particulier, une énergie qu'elles ne soupçonnaient pas. Comme Khady, la plus puissante des trois femmes du recueil de NDiaye, la plus fragile, la plus violentée du recueil, qui garde en elle, malgré tout, la ferme assurance de ce qu'elle est, ces trois femmes ne se laissent pas écraser par les drames qu'elles vivent, elles dévoilent une résistance extrême, doublée d'une dignité inaliénable. 
Dalembert forme véritablement le projet de féminiser cette traversée maritime : les éléments descriptifs des personnages tendent à souligner avec récurrence que leur courage et détermination sont propres à leur sexe et que leurs réactions de solidarité, d'altruisme se nourrissent d'une force ou d'expériences féminines. Ainsi lit-on que Semhar et son amie Meaza "se mirent en route, portées par leur foi et leur détermination de femmes » (DALEMBERT, 2019, p. 134). Réunies par le hasard de la route, Chochana et Semhar se lient d'une amitié solide, sororale, qui leur permet de se confier l'une à l'autre et, dans cet acte où elles " partagent leurs pensées les plus intimes » (DALEMBERT, 2019, p. 166), elles disent à nouveau leur détermination à vivre, à exister comme individus et comme femmes, à laisser une trace de ce qu'elles sont. Elles surmontent ensemble les épreuves de la cale, qui sont agression de " leur humanité et leur féminité » (DALEMBERT, 2019, p. 72). Et si, parfois, elles perdent confiance dans le monde, elles mobilisent les ressources de la religion comme système symbolique de conservation de soi et de dignité, leur foi inaltérable et inébranlable en appelant à la justice et protection divines pour les sortir intactes des épreuves. La séquence finale de la traversée maritime les réunit toutes les trois sur le pont pour l'ultime moment du sauvetage ; toutes trois se tournent vers leur dieu respectif comme si foi et prières constituaient une solide stratégie de défense et de préservation dans le comportement féminin :

La Nigériane psalmodia à voix basse : “ Je lève les yeux vers les montagnes, pour voir d'où me viendra le secours. Mon secours vient de l'Eternel, qui a fait le ciel et la terre... » Les mêmes mots, peu ou prou, que prolongea Semhar : “ L'Eternel est celui qui te garde. [...] L'Eternel gardera ton départ et ton arrivée, Dès maintenant et à jamais. » Au plus fort des cris et de la peur, Dima aussi tourna le regard vers le ciel : "Votre Seigneur est Celui qui fait voguer le vaisseau en mer, pour vous, afin que vous alliez à la recherche de Ses bienfaits. »(DALEMBERT, 2019, p. 296).

Ici, à nouveau, malgré leurs différences, les trois figures féminines semblent étrangement proches et identiques ; la fiction s'enrichit de toutes ses similitudes mais, aussi, des écarts et différences dans le comportement des héroïnes qui sont loin d'être interchangeables. Cette stratégie qui établit de la proximité et des divergences entre les personnages féminins contribue, d'une part, à complexifier le portrait de la femme 
migrante et, d'autre part, à le poser en une sorte de portrait exemplaire et généralisable. Cette même stratégie permet d'identifier ces femmes à la fois comme représentantes de la communauté de candidats à l'exil et comme individualités singulières. C'est dans ce va-et-vient constant que se confirme le caractère hors-norme de ces destins croisés de femmes capables autant de se confondre avec les prisonniers du chalutier en perdition que de s'extraire de la masse anonyme, les “ compagnons de galère » (DALEMBERT, 2019, p. 274), pour les mener à l'assaut de l'écoutille. La mise en valeur de ces personnages féminins, singuliers et exemplaires, provient sans nul doute aussi du choix que fait Dalembert d'inscrire leurs actes et décisions prioritairement dans cette séquence particulière qu'est la traversée maritime.

\section{La traversée maritime comme séquence narrative et argumentative}

A partir d'un large corpus francophone produit des deux côtés de la Méditerranée, Catherine Mazauric remarque, que

La traversée maritime, surtout lorsqu'il s'agit de celle du Détroit, constitue, dans les romans, un épisode isolé. Il s'agit en quelque sorte d'un point culminant du récit, du moment où la destinée des personnages peut basculer, en s'achevant tragiquement par un naufrage, ou déboucher sur une vie nouvelle de l'autre côté de la mer. (2010, p. 156).

Il nous semble surtout que cette étape de l'itinéraire clandestin, tel qu'il est traité par Dalembert, s'élabore comme un topos qui constitue autant une séquence narrative qu'argumentative, séquence fortement identifiable, et suffisamment plastique pour devenir la matrice diégétique de tout le roman.

Mur Méditerranée possède, pour ossature diégétique, la traversée de la mer présentée comme l'étape ultime, celle du " grand jour », et du " non-retour » (DALEMBERT, 2019, p. 17), intervenant chronologiquement après le trajet effectué par voie terrestre ou aérienne par les trois protagonistes. Cette matrice est remplie d'épisodes qui contribuent - par leur traitement narratif et leur mise en perspective - au caractère romanesque du récit du naufrage du chalutier que Dalembert éloigne ainsi d'une parenté avec un documentaire ou un récit journalistique : tempête, mutinerie, assaut de l'écoutille et naufrage 
quand le bateau prend l'eau, enfin le sauvetage difficile et mortel pour beaucoup, sont autant de faits qui augmentent le cauchemar des passagers notamment des " calais » emprisonnés dans le ventre obscur et sans air du navire. L'écriture et la fiction sont également mises au service du romanesque pour traduire avec force détails la terreur des passagers, leurs doutes, leur sentiment d'impuissance et de colère face aux hommes qui leur imposent un traitement inhumain, face aux vents furieux de la tempête qui transforment le bateau en " mixeur " (DALEMBERT, 2019, p. 177), en brindille fragile sur les eaux déchainées. La lutte entre les vagues monstrueuses et le chalutier, étant le plus souvent perçue de l'intérieur de la cale par Chochana et Semhar, est amplifiée par leur imagination et leur peur de mourir. La succession des rebondissements et des oppressions construit une progression du pire : si, en Lybie, Chochana " bascula dans le cauchemar pur et simple » (DALEMBERT, 2019, p. 59) de l'esclavage sexuel entre autres, ici, dans la soute, elle comprend “ que le pire n'avait pas de fond » (DALEMBERT, 2019, p. 64). Cette accumulation de faits, sur lesquels le plus souvent les personnages ne peuvent agir et qui les fait céder au désespoir ou à la résignation, contribue à dramatiser la traversée, voire, à en souligner le caractère tragique dans le sens aristotélicien du terme.

La structure du topos est en outre balisée par les indicateurs temporels dont le but n'est pas seulement de créer un effet de réel : les dates, 16 juillet 2014 à l'initial du roman et 18 juillet 2014, marquent la durée réelle de la traversée, du moment où les trois femmes montent à bord du chalutier jusqu'à leur sauvetage. Néanmoins, la narration va, d'une part, brouiller les repères temporels en se gonflant de la subjectivité qu'autorisent les focalisations internes et, d'autres part, se ramifier grâce aux épisodes que permettent les nombreuses analepses. Dans le premier cas, la narration souligne combien l'expérience trouble l'esprit et la mémoire des personnages qui " ne savaient plus ni quelle heure ni quel jour on était » (DALEMBERT, 2019, p. 14) ou qui ressentent le temps comme dilaté par leur frayeur: "Dans la réalité de la cale d'un bateau en lutte avec une mer démontée, on aurait dit un siècle » (DALEMBERT, 2019, p. 198). Ce jeu sur le temps éprouvé met en lumière le désarroi des personnages livrés à eux-mêmes pour comprendre le désordre du monde ou impulse chez eux une réflexion sur le sens de cette épreuve : 
Au fait, quel jour, quelle date on était? Elle aurait été incapable de le dire. Elle saurait à l'arrivée, pensa-t-elle. Quand elle aurait enfin échappé aux griffes des passeurs. Elle remettrait alors l'horloge de sa vie à l'endroit. Bien sûr, elle ne pourrait pas rattraper les jours, les semaines, les mois perdus dans la quête d'un lendemain meilleur. Mais elle se promit d'en profiter, de faire une de ces javas. Dans cette attente, elle était déterminée à avancer, comme le bateau. A regarder droit devant elle. (DALEMBERT, 2019, p. 96).

Le sentiment de perte du temps raffermit finalement l'héroïne dans son projet d'exil, et les épreuves du trajet se présentent à elle sous la forme d'une parenthèse qui se fermera et dont elle oubliera sans doute un jour toute la violence. Chochana rejoint ici la femme puissante de Marie NDiaye qui, de la même façon, avait la prescience de vivre une étape transitoire de sa vie (2010).

Dans le second cas, la matrice factuelle du topos, -identifiable par les titres des chapitres " Larguez les amarres » et à " A bord du chalutier » -, va accueillir des épisodes antérieurs à la traversée maritime ; ils sont repérables, typographiquement, par les titres également, qui renvoient aux prénoms des trois personnages féminins ainsi mis en valeur. Car il s'agit bien, dans ces séquences analeptiques, d'approcher au plus près de l'histoire de ces femmes et, notamment, de donner à voir les raisons de leur exil. L'auteur nous ramène au point de départ de la quête de l'ailleurs, le moment précis de la prise de " décision » (DALEMBERT, 2019, p. 115, 240). La fuite vers l'Europe suit, certes, des itinéraires différents mais repose également sur des motifs divers : Chochana, la Nigériane, laisse un pays rendu moribond par une sécheresse qui engendre un exode appelé " le mal » (DALEMBERT, 2019, p. 30) ; Semhar quitte l'Erythrée et sa terrible dictature ; Dima, la bourgeoise syrienne habituée à la vie facile, fuit un pays sous les bombes. Dans les trois cas, la décision est mûrie, remplie de volonté et d'espoir pour elles-mêmes et leurs proches, motivée et assumée même si, parfois, les doutes assaillent les personnages en cours de route. Rien d'inconscient ou de suicidaire dans ce choix d'une vie meilleure qui, en outre, place à égalité les motivations politiques et économiques, contrairement aux États européens qui distinguent les réfugiés politiques et les migrants économiques, pour accueillir les premiers et refouler les autres.

La structure du topos offre des possibilités diégétiques qui ouvrent le texte à des va-et-vient temporels et des focalisations féminines 
diverses ; en ce sens, elle est une structure dynamique qui met en relation des vies singulières, qui construit des parallélismes entre des expériences différentes et révèle la complexité de la migration clandestine contemporaine. Si Sylvie Kandé choisissait dans La Quête infinie de l'autre rive (2011) de relater une traversée épique à trois époques différentes, Dalembert opte pour le récit d'une seule traversée vécue en même temps par trois femmes différentes. Ainsi ce n'est pas l'étape ultime qu'il démultiplie pour la rendre épique mais la représentation de ce combat mené par des femmes, bien actuelles, pour s'émanciper d'un destin assigné. Leur force est inscrite dans un présent bien concret et s'ajoute à une forte volonté de dépassement de soi, qui leur permet de se projeter vers un futur à construire. Ce choix de l'auteur haïtien met également en valeur le courage et la détermination de ces êtres qui, même au plus fort de la tempête qui pourrait faire chavirer leur vie, rappellent" qu'ils étaient des humains » et, comme le clame la chétive Semhar, "A ce titre, nous avons droit au respect, comme tous les êtres humains » (DALEMBERT, 2019, p. 274).

Cette matrice textuelle particulière de la traversée maritime, qui met à l'épreuve l'humain, qui est défi à la finitude de la destinée, permet aussi au romancier haïtien d'élaborer un choix d'épisodes et de traitements narratifs qui participent d'un parti pris argumentatif. Certes, l'argumentation est implicite le plus souvent, et prend la forme d'une " dénonciation notifiante " (CHAUDET, 2016, p. 82) mais elle conduit bien le lecteur à prendre conscience du déni d'humanité qu'est la crise migratoire, cette " innommée catastrophe » dont parle Patrick Chamoiseau dans Frères Migrants (2017). Rappelons d'emblée que Dalembert dédie ce roman à Angela Merkel et aux amis de Lampedusa : ces inscriptions liminaires sont à lire comme le souci de prendre part à un débat politique et social qui détermine autant l'accueil ou non des migrants sur le sol européen que leurs conditions d'arrivée. Car, le dessein de l'auteur est de conduire le lecteur vers une prise de conscience de sa propre responsabilité dans ce drame qui se joue en son nom et pour sa sécurité, drame qui serait " la plus grande catastrophe humanitaire de ce début de siècle » (DALEMBERT, 2019, p. 300). Si le romanesque contribue à rapprocher le lecteur des personnages, par l'imagination, la sensibilité et l'émotion, l'agencement de certains éléments diégétiques et les commentaires du narrateur autant que des personnages contribuent également à construire une accusation à l'encontre des États-nations 
et de leurs “ nécropolitiques » (MBEMBE, 2003) qui élèvent murs et frontières pour empêcher le passage et qui violent ainsi un droit de l'homme fondamental. Mur Méditerranée met en exergue ce qu'Achille Mbembe (2018, p. 162) appelle la " frontièrisation » contemporaine : " processus par lequel les puissances de ce monde transforment en permanence certains espaces en lieux infranchissables pour certaines classes de populations [...] multiplication consciente d'espaces de perte et de deuil où la vie de tant de gens jugés indésirables vient se fracasser ». Le récit de cette traversée maritime prend des allures de réquisitoire contre la complicité des autorités et le silence de tous car, “Au fond, qui s'en inquiétait vraiment, à part des ONG dont c'était le fonds de commerce ? " (DALEMBERT, 2019, p. 147). Mais le réquisitoire n'a de validité ici que parce que Dalembert s'efforce de traduire en mots une démarche qui va de la sidération à la considération, selon la formule de Marielle Macé (2017), démarche qui consiste à redonner dignité aux morts et aux vivants. Ainsi le texte n'est-il pas seulement, de façon réductrice, dénonciation véhémente et monument littéraire à la mémoire des morts, il est par-dessous tout (re)connaissance des projets de vie et d'espoirs de ces candidats à l'exil, hommage à leur bravoure qui leur permet de s'inventer un destin ; il proclame enfin l'urgence de reconnaître un “ frère » dans cet Autre en quête d'une Terre jamais promise. Le topos de la traversée maritime se transforme, pour ces raisons, en une traversée poétique qui crée une circulation d'images et d'imaginaires, doublée d'une sédimentation de textes et de discours pour affirmer " Des vies égales » (MACE, 2018, p. 35).

\section{Poétique de la traversée ou traversée poétique}

Dalembert nous convie à une traversée poétique qui est contestation de la frontière devenue "ce lieu zéro de la non-relation et du déni de l'idée même d'une humanité commune, d'une planète, la seule que nous aurions, qu'ensemble nous partagerions, et à laquelle nous lierait notre condition commune de passants » (MBEMBE, 2018, p. 162). La frontière n'est pas un mur qui clôture et empêche le passage, précise Michel Agier; au contraire, elle " met en œuvre à la fois un partage et une relation » (AGIER, 2016, p. 25). La réécriture du topos de la traversée que propose Mur Méditerranée réalise une mise en relation, d'une part, dans les liens que tissent les personnages tout au long du récit et ce, malgré 
ou grâce à leurs différences qui deviennent parfois des complémentarités salutaires, et d'autre part, dans la constellation des échos textuels et du déploiement paradigmatique du sens que ces échos autorisent.

Sur le premier point, nul doute que l'auteur a construit son scénario sur des rapprochements significatifs entre les trois figures féminines qui partagent les mêmes doutes et angoisses, qui trouvent les mêmes ressources en la foi et qui s'épaulent, se renforcent dans les pires moments de la traversée. C'est dans le soutien qu'elles s'apportent mutuellement qu'elles trouvent la force d'avancer et qu'elles évoluent. Dima, plus que les deux autres, finit par agir selon son cœur et son éducation religieuse pour dépasser ses préjugés et son racisme à l'égard des Africaines. Enfin, prisonniers dans le ventre du chalutier, et au cœur de l'enfer de la tempête, les " calais » trouvent, dans la solidarité, la force de se révolter et de partir, tous ensemble, à l'assaut de l'écoutille : " Leurs poumons crachaient 1'espoir, leurs poings nus tapaient, déterminés, contre la trappe. Leurs poings nus cognaient en quête d'air et de liberté [...] Le chant porté par ces centaines de poitrines était une arme létale, plus forte que les flots et les vents. Plus dangereuse que la Méditerranée même » (DALEMBERT, 2019, p. 280-283). Cette force collective - qui est partage d'une même colère et mise en relation du même souffle d'espérance ramène, au-devant du texte, la métaphore du " mur » annoncée dans le titre du roman, mur de la forteresse européenne interdite qui tombera, comme l'enceinte de Jericho.

Le second point apparaît plus significatif encore de cette traversée poétique qui donne à lire le drame de l'exil clandestin comme forme contemporaine de l'exil des peuples opprimés. La trame du roman s'élabore en particulier sur le souvenir de la traite négrière, ce qui ne surprend guère puisque la traversée de l'océan des esclaves africains semble fonctionner comme origine de tout départ dans l'œuvre de Dalembert. Dans L'autre Face de la mer (1998), ce voyage apocalyptique occupait une quinzaine de chapitres en prose poétique dans lequel les esclaves étaient présentés entassés, enchainés, maltraités, privés de tout et surtout d'humanité par leurs bourreaux. Mur Méditerranée ramène à cet épisode douloureux de la cale comme pour mieux rappeler que ce " cheptel » existe encore du fait de la mondialisation : une résurgence, en somme, de l'expansion impérialiste du Vieux continent. Les fuites massives de la jeunesse africaine sont également mises en relation avec l'ensemble des exodes qui essaiment dans les textes religieux. Chaque 
personnage féminin apporte au roman sa part d'évocation religieuse sous forme de prières, de chants religieux, de proverbes, d'épisodes emblématiques des textes fondateurs des trois religions monothéistes. Ainsi, l'exil et l'esclavage des Hébreux sont présents thématiquement dans le récit qui nous mène au fond de la cale du chalutier chargé de migrants clandestins ; ils sont convoqués régulièrement par Chochana qui cherche à reprendre le contrôle de ses nerfs, en récitant la prière du voyageur, ou en chantonnant le Va, pensiero de Verdi ou le psaume "Si je t'oublie Jérusalem » : les paroles du " chœur des esclaves » agissent sur elle, " la déplaçaient en pensée au large de ce cloaque où se consumait son espoir d'une vie meilleure » (DALEMBERT, 2019, p. 195). De même, l'assaut de l'écoutille est présenté comme un Harmaguédon (en référence au combat final de l'Apocalypse) et nombreux sont les épisodes qui rappellent soit la traversée de la mer Rouge qui permet à Moïse de sortir d'Egypte les Hébreux réduits en esclavage par Pharaon (Exode, chapitre 14 et Coran, sourate 26), soit le combat des opprimés pour recouvrer leur liberté. La présence des échos textuels développe explicitement le leitmotiv de l'émancipation dans la foi de dieu, d'une part, et, surtout, la victoire de l'être exilique en général, d'autre part.

La traversée de la mer, moment et espace décisifs dans la migration clandestine puisqu'elle marque le passage sur l'autre rive, la rive interdite, construit une réflexion sur l'exil vécu comme une traversée du désert pour devenir ce que l'on est, aux termes d'épreuves, de dépassement de soi et de victoires. Ce périple clandestin suscite le souvenir de la version apocalyptique de l'exil originaire des esclaves de la traite, " le premier holocauste », tout en s'enrichissant du sens pluriel de l'exode du peuple juif, qui est tout à la fois déportation, arrachement à une terre mais aussi libération et événement fondateur d'Israël. Dalembert ne s'écarte qu'en apparence de sa réflexion habituelle sur l'exil, il en produit une représentation plus ample dans ce jeu de circulation du sens entre ses propres textes et les discours religieux : il démultiplie le sens de l'exil vécu comme déchirement, deuil mais aussi métamorphose de l'être et révélation de soi. Chochana peut dès lors affirmer : "L'essentiel était de garder le contact avec la communauté où qu'ils aillent et de rester fidèles à eux-mêmes » (DALEMBERT, 2019, p. 32) et Dima de préciser : " [...] les humains, c'est pareil aux arbres, ils ne peuvent vivre sans racines. C'est ça qu'on tient dans cette grande aventure qu'est la vie. Qu'on arrive à partir même très loin et revenir sans se perdre. Sinon, 
on dessèche sur pied jusqu'à se consumer » (DALEMBERT, 2019, p. 255). La migration contemporaine révèle à nouveau que c'est dans le chemin que l'individu se construit, qu'il avance en regardant derrière lui, dans son " pays-temps » originaire, tout en se projetant au loin, se reconnaissant ainsi, " étranger et voyageur sur la terre » (selon le Livre de l'Exode) toujours en marche, jamais arrivé.

Mur Méditerranée trouve sa place dans la série de " romans à idées » de Dalembert (PAGEAUX, 2018, p. 18) : il dénonce la violence des frontières et des assignations dans un lieu unique et une identité figée. L'auteur retrace avec minutie une odyssée féminine car les femmes subissent plus que d'autres la violence de ce déni d'humanité qu'est la traversée maritime interdite pour atteindre la rive européenne. L'actualité de la crise migratoire permet à Dalembert de désancrer sa fiction et sa réflexion, de son île natale, pour aller écouter les voix de ce " bateaumonde » de la mondialisation. Les voix de ces hommes et femmes partis à l'assaut du “ mur » disent l'exil comme traumatisme, comme oppression mais aussi comme enraciné en l'homme. Leur traversée de la mer déchainée, qui, aujourd'hui, a cessé d'être mare nostrum, devient topos transculturel qui accueille les échos des autres traversées collectives, sous formes de chants, légendes, discours littéraires et religieux, ceux qui ont marqué l'histoire universelle et définissent l'homme dans son essence exilique.

\section{Références}

AGIER, M. Les Migrants et nous : Comprendre Babel. Paris : CNRS, 2016. DOI : https://doi.org/10.3917/cnrs.agie.2016.01.

CHAMOISEAU, P. Frères Migrants. Paris : Éditions Du Seuil, 2017.

CHAUDET, C. Ecritures de l'engagement par temps de mondialisation. Paris : Classiques Garnier, 2016.

DALEMBERT, L-P. Et le Soleil se souvient... Paris : Éditions L'Harmattan, 1986.

DALEMBERT, L-P. L'autre Face de la mer. Paris : Stock, 1998.

DALEMBERT, L-P. L'Île au bout des rêves. Paris : Du Rocher, 2007. 
DALEMBERT, L-P. Louis-Philippe : “ Je suis né féministe ». Midi Libre, Saint-Jean-de-Védas, 21 nov. 2019. Entretien donné à Stephane Barbier. Disponible sur le site : https://www.midilibre.fr/2019/11/20/ louis-philippe-dalembert-aragon-disait-que-la-femme-est-lavenir-delhomme-jai-envie-de-dire-quelle-est-lavenir-de-lhumanite,8552411.php Consulté le : 17 avril 2020.

DALEMBERT, L-P. Mur Méditerranée. Paris : Sabine Wespieser Éditeur, 2019.

DALEMBERT, L-P. Noires Blessures. Paris : Mercure de France, 2010.

DIARD, D. Entre l'ici et l'ailleurs : Luis-Philippe Dalembert l'aède vagabond. Loxias-Colloques, Nice, janv. 2018. Disponible sur le site : http ://revel.unice.fr/symposia/actel/index.html ?id=1017. Consulté le : 12 oct. 2019.

DIOME, F. Celles qui attendent. Paris : Flammarion, 2010.

DIOME, F. Le Ventre de l'Atlantique. Paris : Anne Carrière, 2003.

GHINELLI, P. Archipels littéraires. Montréal : Mémoire d'Encrier, 2005.

KANDE, S. La Quête infinie de l'autre rive. Paris : Gallimard, 2011.

MACE, M. Des vies égales. In : Au Cour de l'errance. Montpellier : Chèvre-Feuille Etoilée, 2018, v. 73-74. p. 33-36.

MACE, M. Sidérer, considérer : Migrants en France. Paris : Verdier, 2017.

MARI, J-C. Les Bateaux ivres : L'odyssée des migrants en Méditerranée. Paris : J-C Lattés, 2015.

MAZAURIC, C. Mobilités d'Afrique en Europe, récits et figures de l'aventure. Paris : Karthala, 2012. DOI : https://doi.org/10.3917/kart. mazau.2012.01.

MAZAURIC, C. Voyages au féminin et subjectivations transafricaines. In : CANUT, C, MAZAURIC, C. La Migration prise aux mots : Mise en récits et en images des migrations transafricaines. Paris : Le Cavalier Bleu, 2014. p. 47-61.

MBEMBE, A. Le grand débarras. I $n$ : CHAMOISEAU, P. ; LE BRIS M. Osons la fraternité ? Les écrivains aux côtés des migrants. Paris : Éd. Philippe Rey, 2018. p. 157-169. 
MBEMBE, A. Necropolitics. Public Culture, Durham, v. 15, issue 1, p. 11-40, Jan. 2003. DOI : https://doi.org/10.1215/08992363-15-1-11.

NDIAYE, M. Trois Femmes puissantes. Paris : Gallimard, 2010.

NOUSS, A. La Condition de l'exilé. Paris : Édition de la Maison des Sciences de l'Homme, 2015. DOI : https://doi.org/10.4000/books. editionsmsh.5919.

PAGEAUX, D. H. Le Roman selon Louis-Philippe Dalembert : entre 'réalisme poétique' et 'néo-baroque'. Loxias-Colloques, Nice, janv. 2018. Disponible sur le site : http ://revel.unice.fr/symposia/actel/index. html ?id=1019. Consulté le : 12 oct. 2019.

VIGNOLI, A. Louis-Philippe Dalembert, vagabond jusqu'au bout de la fatigue. Il Tolomeo, Venezia, v. 18, p. 29-40, dic. 2016.

WEIL, M. Avant-propos. In : FERRAND, N.; WEIL, M. Homo narrativus: Recherches sur la topique romanesque dans les fictions de langue française avant 1800. Montpellier : Presses universitaires de la Méditerranée, 2001. Disponible sur le site : http ://books.openedition. org/pulm/1307. Consulté le : 31 août 2020.

Recebido em : 30 de junho de 2020.

Aprovado em : 7 de outubro de 2020. 